\title{
Service Inputs and Costs of Care Related to Outcomes Among Cognitively Impaired Nursing Home Residents
}

\author{
Douglas Holmes ${ }^{1,2 *}$, Jeanne Teresi ${ }^{1,3}$ and Jian Kong ${ }^{1}$ \\ ${ }^{1}$ Research Division of the Hebrew Home for the Aged at Riverdale, USA ${ }^{2}$ Columbia University Faculty of Medicine, USA ${ }^{3}$ Columbia University Stroud \\ Center, New York State Psychiatric Institute, USA
}

\begin{abstract}
Background: There are over 17000 nursing homes in the United States. Within these, special care units (SCUs) provide a separate residential and/or activity locus for residents, and are expected to provide more staff time and more specialized staff assignments. This paper addresses a fundamental issue relating to the nature, quality and quantity of resident care inputs: what impacts of SCUs are associated with added service inputs, and thus with personnel costs, recognizing that personnel account for the majority of costs associated with nursing home care?

Aims of the study: The aim of this aspect of the study was to determine the extent to which additions of staff would result in a diminution of deviant behaviors among residents of special care and of traditional care units.

Method: The data were collected from a random sample of ten downstate nursing homes located in New York State. Using rigorous sampling procedures, random samples of 40 residents were drawn from each of the facilities, equally divided between special care unit and traditional care unit residents. Thus, the sampling design involved two levels of clustering: subjects were clustered within units and units were clustered within facilities. The observational behaviour measure was taken from the INCARE (institutional version of the Comprehensive Assessment and Referral Evaluation). The behavioral observation measure contains 23 items such as 'disruptive of others', 'picks/pulls clothing', 'repetitive movements', 'repetitive questioning' and 'wandering'. Each item is rated as to frequency of occurrence; ratings are collected on three occasions, and averaged. Outcome and covariate data (e.g., behavior and cognition) were collected by trained research staff who visited each site for three to four weeks of intensive data collection, accomplished through direct resident interviews, staff interviews and questionnaires and chart data abstraction. The clinical staff time data were collected using the InfoAide system, whereby each care provider used a portable barcode scanner to record the type of care given, the recipient and the duration of care.

A random effects model using the SAS mixed procedure was applied to the data; adhering to this model, some effects were fixed and some random. The random effects were comprised of the subject (intercept or subject starting point at baseline) and the
\end{abstract}

*Correspondence to: Douglas Holmes, Director, National Alzheimer Center, 5901 Pausade Avenue, Riverdale, NY 10471, USA.

Source of funding: The data on which this paper is based were collected with the support of two grants from the National Institute on Aging: AG10330 and RO1 AGO8948. unit; used here was restricted maximum likelihood (REML) with the EM algorithm.

Results: There was a significant reduction of behavior disorder associated with more provision of aide time in SCUs as contrasted with non-SCUs. The greater the service provided, the greater the slope, i.e., the greater the reduction. That is, while SCU residents showed improvements in behavior accompanying increases in aide time, no such change was observed among non-SCU residents. Implications for Health Care Provision and Use: The significant effect of SCU $\times$ time $\times$ aide-minutes indicates that more aide time on SCUs was associated with improvement in behavior. It appears that the important ingredient in relation to the reduction of behavior disorder is not membership in an SCU per se, but the provision of more aide time within SCUs. Those SCUs that provide more aide time have a better behavior outcome. Thus, these findings point to additional paths for exploration, i.e., future research needs to focus on elements of SCUs such as the available amounts of program and staff resources rather than on SCU status alone. (C) 2000 John Wiley \& Sons, Ltd.

Received 5 July 2000; accepted 18 October 2000

\section{Background}

There are over 17000 nursing homes in the United States (US); ${ }^{1}$ estimates suggest that as many as $90 \%$ of their 1.5 million elderly residents suffer from cognitive impairment, ${ }^{2,3}$ and $70-80 \%$ from dementing illness. ${ }^{4,5}$ A growing proportion (approximately 20\%) of US nursing homes maintain special care units (SCUs), which, in principle, are specifically targeted to the needs of persons with dementing illness. ${ }^{6}$ SCUs provide a separate residential and/or activity locus for these residents, and are expected to provide more staff time and more specialized staff assignments; as a matter of fact, the majority of definitional criteria usually applied in distinguishing between SCU and traditional care are staff related. ${ }^{7}$ The provision of additional staff has far-reaching implications, e.g., for hiring, training, monitoring and, from a managerial perspective, for additional cost. It has been estimated that the costs of direct-care personnel amount to perhaps $80 \%$ of the average nursing home's total budget ${ }^{8-10}$ and nurse aides constitute the largest category of direct service provision. ${ }^{11,12}$ Moreover, Mehr and Fries ${ }^{13}$ and Fries 
et $a l .{ }^{14}$ have noted that staff time expenditures constitute the largest component of cost of care that relates directly to the characteristics of individual residents. Also important from a managerial perspective, staff inputs are the most immediately malleable major component of nursing home care: staff ratios, assignments and patterns can be changed, overnight, at will.

This paper addresses a fundamental issue relating to the nature, quality and quantity of resident care inputs: what impacts of SCUs are associated with added service inputs, and thus with personnel costs? The aim of the study was to determine the extent to which addition of staff would result in a diminution of deviant behavior among residents of special care and of traditional units. One hypothesis is that SCU status and the interaction of SCU and minutes of care provided by aides will result in reduction of behavior disorder.

Most frequently, service data are available in the form of (a) administrative records (e.g., numbers of staff), (b) direct observations (e.g., time and motion studies usually conducted by trained observers with time recording devices), (c) work sampling, in which random work occasions are timed, (d) diaries or logs (maintained by the service providers) or (e) retrospective recall by key informants. Despite the availability of these approaches, the collection of individual resident service data has remained problematic because fundamental measurement requirements are not fulfilled. ${ }^{15-18}$ Interrater and test-retest reliability are often poor, resulting in questionable validity of the data produced by such methods. (For a complete discussion of the relative merits of the different approaches, see Holmes et al. ${ }^{19}$ ) For the current study, a bar coding system (InfoAide $($ ) ) was used, through which the service provider recorded the ID of the service recipient, the nature of the service provided, and the duration of service provision (in addition to the identity of the provider), all with a sweep of the datawand, a recording device. Because barcode sweeping becomes part of the service-providing act, InfoAide is less prone to the biases associated with other techniques for data collection. Moreover, internal monitoring routines self-identify occasions in which an unrecorded action is likely to have occurred. This system is described in greater detail in a recent article which also presents data in support of the system's validity. ${ }^{20}$

\section{Method of Procedure}

\section{Sample}

Staff-barcoded service data were collected with respect to a sample of residents from a total of ten randomly selected nursing homes (five with and five without SCUs), located in downstate New York. In facilities with an SCU, a sample of approximately 20 residents was taken from the SCU and measures of cognitive and functional impairment were collected by trained staff interviewers. The average $\mathrm{MMSE}^{21}$ score was calculated for the SCU sample and used to select that traditional unit in the facility which best matched the level of cognitive impairment found on the SCU. This always turned out to be the unit with the most cognitively impaired residents. Twenty residents then were selected randomly from the traditional unit, yielding a total of 40 residents from each facility. For ease of implementation, the barcoded service information was collected not on just the samples, but on all residents of both units. In facilities without an SCU, the average MMSE score for each unit was examined and the two units most similar to SCUs in terms of levels of cognitive impairment, i.e., whose residents had the highest average cognitive impairment scores as measured by the MMSE, were chosen for the staff input study. Twenty residents were selected at random from each study unit (total $n=40$ per facility). In all cases, random selection (first of facilities, then of residents within facilities) was accomplished through application of a pseudo-random selection program offered as part of the SPSS software package. $^{22}$

It was possible to collect staff data and accompanying complete personal care data for a total of 336 of the 400 selected residents, reflecting an overall response rate of $84 \%$. 'Non-participants' included residents whose primary language was other than English or Spanish, who were too physically ill to be interviewed and/or who had died or been transferred to a hospital prior to collection of all study data. Among the 336 participants, 225 were in traditional units and 111 in SCUs. SCU residents were slightly older (85.4 years versus 82.4 years, $p<0.05$ ), and more cognitively impaired (pro-rated $\mathrm{MMSE}^{21}$ indicating, on average, 'severe' versus 'moderate' dementia, $p<0.01$ ). There were no significant differences between SCU and non-SCU cohorts in terms of ADL functioning (both were, on average, dependent with an intermediate level of impairment), and both groups included approximately $70 \%$ females.

\section{Measures}

\section{Outcomes and Covariates}

Outcome and covariate data (e.g., behavior and cognition) were collected by trained research staff, who visited each site for three to four weeks of intensive data collection, accomplished through direct resident interviews, staff interviews and questionnaires and chart data abstraction. Some of the study measures had been developed, adapted or adopted as part of the common-core measurement protocol developed for the NIA cooperative studies of dementia care, ${ }^{23}$ others were well known assessments, such as the Mini Mental Status Exam (MMSE). ${ }^{21}$ For this sample the internal consistency of the MMSE was 0.83, estimated using Cronbach's alpha. An index of comorbidity was constructed by summing medical conditions abstracted from the chart. The observed range was from 0 to 7 .

The observational behavior measure was taken from the INCARE (institutional version of the Comprehensive Assessment and Referral Evaluation) developed during a series of cross-national studies conducted in the 1970s. ${ }^{24-27}$ The behavioral observation measure contains 23 items such as 'disruptive of others', 'picks/pulls clothing', 'repetitive 
movements', 'repetitive questioning' and 'wandering'. Each item is rated as to frequency of occurrence; ratings are collected on three occasions within a two-day period and averaged. Thus, one summary measure of behavior was available for each wave of data collected. The distribution of emergent scores was from 0 to 19 ; interrater reliabilities for this sample were greater than 0.90 .

\section{Service Use and Cost}

In view of problems (sources of bias) associated with traditional methods for collecting staff time input data, the author had previously developed (with support from the Health Care Financing Administration and the National Institute on Aging) a system ('InfoAide(C) ) with which each service provider could record automatically the amount of time (subsequently monetized) spent providing each of a list of services to specific residents. ${ }^{28,29}$ In brief, InfoAide generates and uses barcoded service sheets which, in conjunction with a portable barcode reader and accompanying database management system records and generates data on what is provided, by whom, to whom, for how long. Each service category is accompanied by two bar codes, one appearing in a 'start' column, the second appearing in the 'finish' column. In the present application, the service provider logged his/her ID number into the portable scanner at the beginning of the shift, and then recorded each occasion of service delivery by sweeping the resident ID barcode, followed by the particular service 'start' code or 'finish' code, as the case may be (the datawand automatically recorded the data and time of each sweep). At the end of the shift, these data were downloaded to a laptop computer for processing using the InfoAid database management system.

\section{Statistical Approach}

A random effects model using the mixed procedure ${ }^{30}$ was applied to the data; adhering to this model, some effects were fixed and some random. The random effects were comprised of the subject (intercept or subject starting point at baseline) and the unit. Modeling the intercept as random controls somewhat for the bias associated with individuals entering the study at different levels of disorder. Additionally, random effects models allow modeling of different rates of change over time (rather than a constant rate of change) and for modeling the covariance structure (rather than just the mean) over time. While other models allow slopes and intercepts to vary among individuals, they stipulate that these remain constant over time.

There are many methods for modeling the covariance structure (e.g., compound symmetry, autoregressive); shown below are the models tested. There are several methods of estimating coefficients. Used here was restricted maximum likelihood (REML) using the EM algorithm. The model assumes a normally distributed dependent variable (this assumption was checked, and transformations performed as necessary). The model also assumes that data are missing at random. This assumption is necessary for the internal imputations, which allow use of the entire baseline data set.
The analysis used only two time points (baseline and sixmonth follow-up) because of the relatively great attrition that had occurred by the third wave of data collection. For example, while the attrition from the first to the second was only $11 \%$, attrition between baseline and the second wave was $54 \%$ (baseline $n=325$, time $1 n=288$ and time 2 $n=149$ ). Because most attrition was due to death, and mortality is related to cognitive impairment and behavior disorder (the outcome variable), it is likely that the data were not missing at random at the time of the second follow-up.

The sampling design involved two levels of clustering: subjects were clustered within units and units were clustered within facilities. In modeling the covariance structure, it first must be determined whether repeated observations are correlated in a way that depends on time between observations (here, time is considered continuously as 'months since baseline' because, although the observations were approximately six months apart, the range was from 2.5 to 9 months). Second, respondents from the same unit may have different effects depending on the type of unit (SCU or non-SCU); finally values from subjects from SCUs and from non-SCUs in the same facility might be associated. Thus it was necessary to model the covariance due to repeated measurements on subjects, to residence in the same unit, and to residence in the same facility.

Akaike's information criteria (AIC) and Schwartz's Bayesian criteria (SBC) were used to determine the most appropriate covariance structure. A random-effects model using SAS PROC MIXED ${ }^{31}$ was used. Preliminary analyses showed that there was no need to model the clustering due to units within facilities, and that the variances for SCU and non-SCUs were homogeneous.

\section{Results}

\section{Modeling the Covariance Structure}

First, the clustering due to repeated measures was modeled. Shown below are the values for AIC and SBC.

As shown, $\mathrm{Sp}$ (power) constitutes the best model; this model is:

$$
\Sigma_{\mathrm{ij}}=\sigma^{2} \rho_{\mathrm{ij}}^{\text {month }}, \text { where } \sigma^{2}=0.4870 \text { and } \rho=0.6685
$$

where $\rho$ is the correlation between baseline and first followup for unit level of time (one month), and where $\sigma^{2}$ is the residual. The correlation between repeated measures decreases over time at an exponential rate. This indicates

\begin{tabular}{lll}
$\begin{array}{l}\text { Covariance structures for } \\
\text { repeated measures }\end{array}$ & AIC & SBC \\
\hline Compound symmetry & 624.1 & 627.9 \\
Unstructured & 624.8 & 630.5 \\
Autoregressive & 624.1 & 627.9 \\
Sp(power for month) & 623.5 & 627.3 \\
\hline
\end{tabular}


that the more proximal repeated measures are more highly correlated than are distal measures. When the random effect due to the unit is added, the model improves:

\begin{tabular}{lll}
\hline & AIC & SBC \\
\hline Sp(power), random on unit & 620.3 & 624.5 \\
\hline
\end{tabular}

\section{Modeling the Measurements}

Shown in Table $\mathbf{1}$ are the baseline characteristics of SCU and non-SCU residents in terms of the variables included

Table 1. Means and standard deviations for SCU and non-SCU residents on baseline variables.

\begin{tabular}{|c|c|c|}
\hline Variable & $\operatorname{SCU}(n=111)$ & Non-SCU $(n=214)$ \\
\hline $\begin{array}{l}\text { Observation of } \\
\text { behavior } \\
\text { Disorder (log } \\
\text { transformed) }\end{array}$ & $1.12(0.89)^{*}$ & $0.87(0.82)$ \\
\hline $\begin{array}{l}\text { Original non- } \\
\text { transformed }\end{array}$ & $3.40(3.71)$ & $2.31(2.84)$ \\
\hline Age & $85.44(7.60)^{*}$ & $82.41(11.45)$ \\
\hline Comorbidity & $1.61(1.53)^{*}$ & $2.36(1.43)$ \\
\hline $\begin{array}{l}\text { Centered Cognitive } \\
\text { Disorder }\end{array}$ & $0.19(0.89)^{*}$ & $-0.15(1.02)$ \\
\hline (MMSE) & $\begin{array}{l}\text { (severe to extreme } \\
\text { cognitive } \\
\text { impairment) }\end{array}$ & $\begin{array}{l}\text { (moderate to severe } \\
\text { cognitive } \\
\text { impairment) }\end{array}$ \\
\hline $\begin{array}{l}\text { Log-transformed costs } \\
\text { of medical doctor } \\
\text { time }\end{array}$ & $0.29(0.74)$ & $0.39(0.90)$ \\
\hline $\begin{array}{l}\text { Log-transformed costs } \\
\text { of physical therapy } \\
\text { time }\end{array}$ & $0.10(0.44)^{*}$ & $0.34(0.72)$ \\
\hline Time spent by aides & $49.82(21.11)^{*}$ & $41.51(18.72)$ \\
\hline
\end{tabular}

*Significant at the .05 level. in the model. In addition to the variables listed below, a quadratic term involving the MMSE was also entered in the model to account for the non-linear relationship of MMSE scores with behavior disorder. At low and at very high levels of cognitive disorder behavior disorder is less. Several variables were $\log$ transformed for purposes of normalization; these were the behavior disorder outcome and the two cost variables associated with medical doctor (MD) and physical therapy (PT) time. The cognitive disorder variable, the Mini-Mental Status Exam (MMSE), ${ }^{21}$ was centered around their average values (transformed to a standard $Z$ score) to avoid collinearity between the interaction and quadratic terms and the original term, all of which contained the MMSE. Finally models were checked to ensure that there was no collinearity.

As shown in Table 1, as contrasted with non-SCU residents SCU residents were significantly older, more behavior disordered and cognitively impaired, and received more aide time; however non-SCU residents had significantly higher costs of physical therapy care, perhaps because they were generally more physically ill, with more comorbid conditions. Only variables which differentiated significantly between SCU and non-SCU residents at baseline were entered into the final model.

Shown in Table 2 are the zero-order correlations among variables included in the model.

As shown, behavior disorder was related to cognitive impairment, to amount of time received from nurse aides, and to being a resident of an SCU. Behavior disorder was significantly negatively correlated with comorbidity and with costs of physical therapy. As expected, cognitive impairment was also associated with residence in an SCU and with receipt of more aide time. Residents of SCUs received significantly more aide time at baseline, but not at follow-up.

Within both SCUs and non-SCUs, behavior disorder was significantly associated ( $r=0.28$ for SCUs and 0.20 for nonSCUs) with more aide time at baseline. The relationships remained significant, but weaker at follow-up.

The distribution for minutes of aide service time per resident is shown below:

$\begin{array}{rlr}0-20 & \text { minutes/day } & 13 \% \\ 20-43 & \text { minutes/day } & 43 \%\end{array}$

Table 2. Correlations among study variables at baseline (below diagonal) and follow-up (above diagonal). (Correlations along the diagonal are those between baseline and follow-up.)

\begin{tabular}{|c|c|c|c|c|c|c|c|c|}
\hline & Behavior & Age & Comorbidity & MMSE & Aide time & SCU & Medical cost & PT cost \\
\hline Behavior & $0.21 * *$ & $0.12 *$ & -0.04 & $0.42 * *$ & $0.19 * *$ & $0.14 *$ & -0.03 & -0.08 \\
\hline Age & 0.04 & 1.00 & $0.15 *$ & $0.21 * *$ & -0.01 & $0.14 *$ & $-0.26 * *$ & -0.08 \\
\hline Comorbidity & $-0.13 *$ & $0.16 * *$ & $0.78 * *$ & $-0.15^{*}$ & -0.01 & $-0.20 * *$ & -0.03 & 0.11 \\
\hline MMSE & $0.48 * *$ & $0.22 * *$ & $-0.15^{*}$ & $0.84 * *$ & $0.40 * *$ & $0.27 * *$ & -0.11 & -0.03 \\
\hline Aide time & $0.26 * *$ & 0.07 & 0.05 & $0.45 * *$ & $0.58 * *$ & 0.07 & -0.06 & $0.16^{* *}$ \\
\hline SCU & $0.16^{* *}$ & $0.14 *$ & $-0.24 * *$ & $0.16 * *$ & $0.20 * *$ & 10.00 & $-0.19 * *$ & $-0.22 * *$ \\
\hline Medical cost & -0.02 & $-0.19 * *$ & -0.10 & 0.03 & 0.04 & -0.06 & $0.68 * *$ & $0.14 *$ \\
\hline PT cost & $-0.14 *$ & 0.02 & $0.16^{* *}$ & -0.09 & -0.03 & $-0.17 * *$ & -0.04 & $0.39 * *$ \\
\hline
\end{tabular}

${ }^{*} p<.05$.

$* * p<.01$. 
$43-60$ minutes/day $\quad 24 \%$

$60-80$ minutes/day $\quad 16 \%$

80-117 minutes/day $\quad 4 \%$

Shown in Table 3 is the solution for the fixed effects.

Table 3. Mixed model solution for fixed effects

\begin{tabular}{lc}
\hline Variable & Coefficient \\
\hline Intercept & $0.972^{* *}$ \\
Age & -0.003 \\
Comorbidity & -0.013 \\
MMSE & $0.375^{* * *}$ \\
Aide time & $0.005^{* *}$ \\
SCU & $0.243^{*}$ \\
Medical cost & -0.029 \\
PT cost & $-0.088^{\mathrm{t}}$ \\
MMSE $\times$ SCU & $0.167^{*}$ \\
MMSE $\times$ SCU & $-0.218^{*}$ \\
Aide time $\times$ SCU $\times$ time & $-0.001^{*}$ \\
\hline
\end{tabular}

${ }^{t} p<.10 ; * p<.05 ; * * p<.01 ; * * * p<.001$.

Using transformed behavior scores adjusted for all covariates, the following is observed.

Controlling for MMSE, comorbidity, age, aide minutes, MD cost and PT cost, SCU residents were more behavior disordered at baseline. Because subjects did not constitute a new admission cohort, and because SCUs frequently target cognitively impaired individuals with behavior disorder, the SCU residents began with more behavior disorder than did their matched non-SCU counterparts. This finding is reflected in the estimate of the intercept shown in Table 3 (for nonSCU residents, it is 1.0236 while for SCU residents it is $1.0236+0.2539)$ - indicating significantly greater adjusted baseline values for SCU residents).

Excluding the non-significant terms from the model, behavior $=\exp [0.7547+0.3586 \mathrm{mmse}+0.0043$ aide time -0.1006 pt cost $+0.2462 \mathrm{SCU}+0.1821 \mathrm{mmse} \mathrm{SCU}$ $-0.2334 \mathrm{mmse}^{2} \mathrm{SCU}-0.0008$ time aide time SCU] -1 .

These results indicate that cognitive disorder and membership in an SCU were associated with baseline behavior disorder, as was more aide time and less PT cost. More aide time provided in SCUs (as contrasted with that provided in nonSCUs) was associated with reduction in behavior disorder over time.

The dependent variable was log transformed so the coefficients cannot be interpreted directly; therefore, the scores were translated back to their original units of measurement, and S-Plus was used to graph the results in order to interpret the trend between behavior and time for SCUs and non-SCUs. Shown in the attached graphs (see Figures 1 and 2) are examples of the effects. The S-Plus graph shows that an SCU-resident, as contrasted with a non-SCU resident, receiving an average amount of aide care time decreased about 1.3 points per year in terms of behavior disorder scores.

There was an interesting relationship between cognitive disorder and behavior disorder. For non-SCUs the relationship appears to be linear, while it is curvilinear for SCU residents (for which there was a significant quadratic term). With higher cognitive impairment levels, there appears to be little difference between SCUs and non-SCUs in terms of observed behavior disorder. (See Figure 3.) Perhaps this indicates that targeting mild to moderate cognitive impairment for treatment or placement on SCUs reflects rational planning.

Non-SCU residents did not change over time in terms of behavior disorder, but there was an association between aide time and behavior disorder at baseline. Those with more impairment in behavior disorder received more minutes of aide time.

Obbehpr by Time (Aidetot=43 the average)

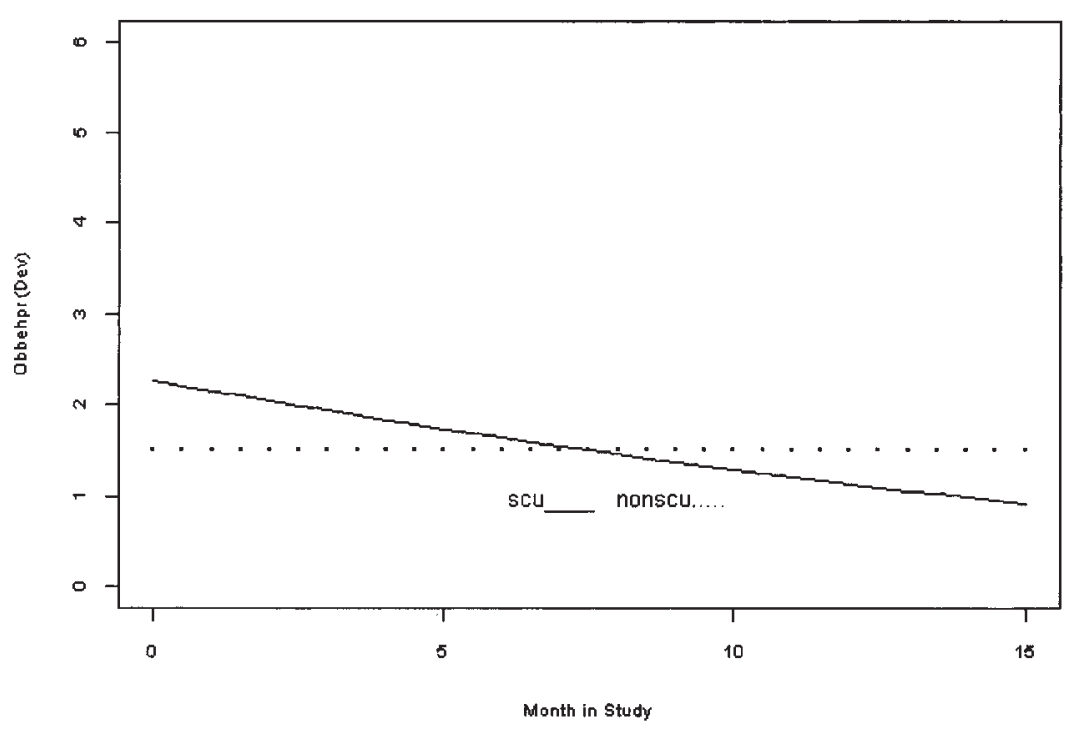

Figure 1. Observed behavior by time (aidetot $=43$ average): only significant terms included. 

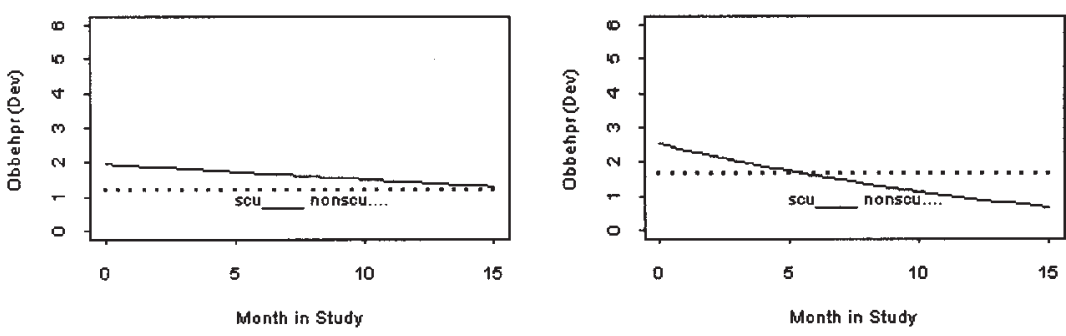

Obbehpr by Time (Aidetot=80)

Obbehpr by Time (Aidetot=117)
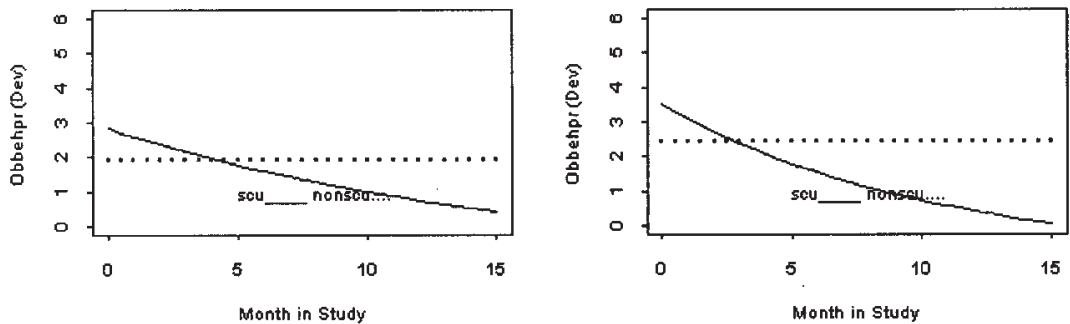

Figure 2. Observed behaviour by time (different level of aidetot): only significant terms included.

Obbehpr by mmse

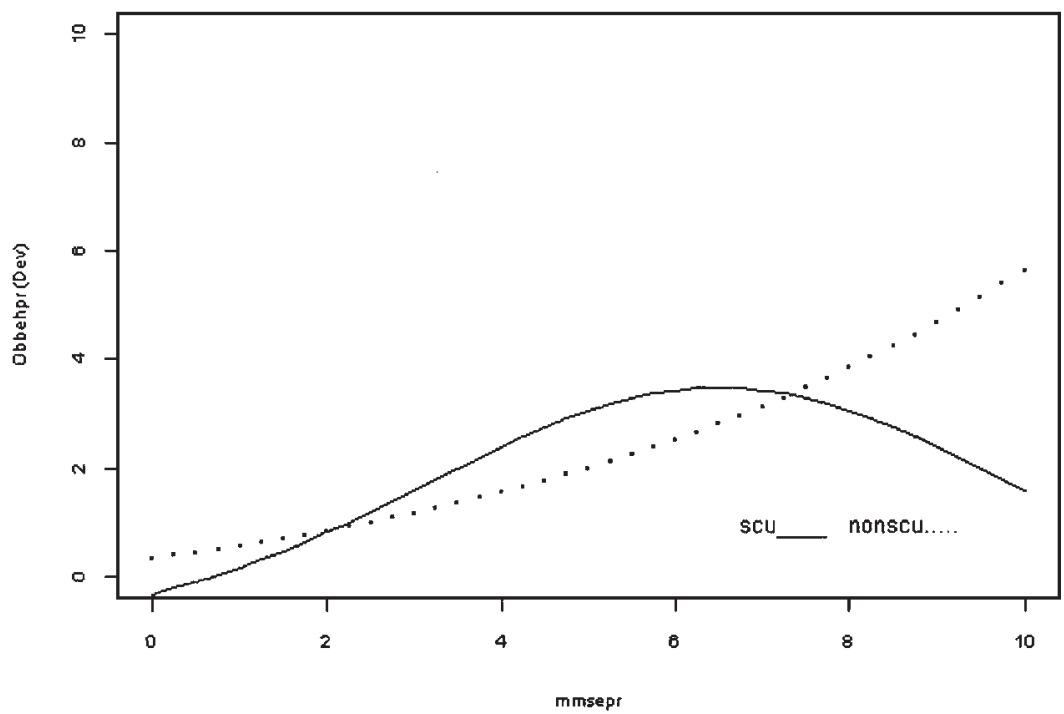

Figure 3. Observed behavior by MMSE (baseline).

\section{Discussions}

There was a significant reduction of behavior disorder associated with more provision of aide time in SCUs as contrasted with non-SCUs. The greater the service provided, the greater the slope, i.e., the greater the reduction. For example, for aide time equal to 20 minutes, the slope indicates a reduction of about 0.8 points per year; for aide time of 43 (about the average amount of service provided), the reduction in behavior disorder was about 1.3 points per years; for aide time of one hour about 1.8 points per year; for aide time of 80 minutes, about 2.5 points per year; finally for provision of the highest amount of time (117 minutes), the reduction was about 3 points per year. While behavior disorder remained more or less constant in the non-SCUs at all levels of nursing aide input, relatively small incremental service use and reimbursement for aide service is accomplished by relatively robust positive changes in behavior among SCU residents. Translated into costs, using the local average reimbursement rate for aides $(\$ 17.00 / \mathrm{hr}$, including fringes), in those units (SCUs) where only $\$ 6.46 /$ day (as contrasted with the average expenditures rate of $\$ 12.18 /$ day) was spent for aides per resident day, deviant behavior still decreased, over 15 months, to the (constant) level of disorder found among the non-SCU residents. However, if $\$ 17 /$ day was spent for aides per resident day (an increase of under $\$ 5$ over the average), the accompanying deviant behaviors decreased substantially over the 15 study 
months. An increase of $\$ 10$ aide time per resident per day was accompanied by further decrease in behavior disorder (which occurred sooner). However, further increase yielded only slightly greater reductions in deviant behavior. All of these data indicate that a relatively modest incremental cost input is associated with significant and meaningful reductions in deviant behaviors, so long as this occurs in a special care environment. The data also indicate, however, that equivalent incremental expenditures have little or no effect in non-SCUs and that, even in SCUs, a state of equilibrium is reached at which further incremental expenditures can be expected to have little result.

It is of importance that there is a significant effect in non-common core measures of affect and behavior (observations), although other analyses with less sensitive measures did not show much effect. This is similar to the findings of van Haitsma and colleagues (2000), ${ }^{32}$ who, using observational measures, report effects associated with SCU status.

In summary, preliminary analyses had shown that there was a non-significant effect of SCU membership (SCU $\times$ time) on change in behavior. The fact that there is a significant effect of SCU $\times$ time $\times$ aide minutes indicates that more aide time on SCUs was associated with improvement in behavior. It appears that the important ingredient in relation to the reduction of behavior disorder is not membership of an SCU per se, but the provision of more aide time within SCUs. Those SCUs that provide more aide time have a better behavior outcome. Future research needs to focus on elements of SCUs such as amount of program and staff resources rather than SCU status alone.

\section{References}

1. NCHS. Use of nursing homes by the elderly: preliminary data from the 1997 National Nursing Home Survey. NCHS Advanced Data No. 312. US Public Health Service, 2000; 1.

2. Teresi J, Morris J, Mattis S, Reisberg B. Cognitive impairment among SCU and non-SCU residents in the United States: estimates from the National Institute on Aging Collaborative Studies of Dementia Special Care units for Alzheimer's disease. Res Practice Alzheimer's Dis 2000; 4: 117-138.

3. Teresi J, Evans D. Cognitive assessment measures for chronic care populations. J Mental Health Aging 1996; 2: 151-174.

4. Maslow K. Current knowledge about special care units: Findings of a study by the U.S. Office of Technology Assessment. Alzheimer's Dis Assoc Disorders Int J 1994; 8: 14-40.

5. Rovner W, Rabins PV. Mental illness among nursing home patients. Hospital Community Psychiatry 1985; 36: 119-128.

6. Leon J, Cheng C-K, Alvarez RJ. Trends in special care: Changes in SCU from 1991 to 1995 ('95/96 TSC). J Mental Health Aging 1998; 149-168.

7. Holmes D, Splaine M, Teresi J, Ory M, Monaco C, Ramirez M, Barrett V. What makes special care special: Concept Mapping as a definitional tool. Alzheimer's Dis Assoc Disorders Int J 1994; 8 (suppl. 1): S41-S53.

8. Palmer H, Cotterill P. Studies of nursing home costs. In: Vogel R, Palmer $\mathrm{H}$ (eds). DHHS, Health Care Financing Administration: Long Term Care: Perspectives from Research and Demonstrations, Washington, DC, 1982; 665-722.

9. Glandon G, Lindeman D, Holmes D. Issues in measuring costs in institutional settings. J Mental Health Aging 1997; 3 (1): 129-144.
10. Holmes D, Teresi J, Lindeman D, Glandon G. Measurement of personal care inputs in chronic care settings. $J$ Mental Health Aging 1997; 3 (1): 119-127.

11. Wright L. A reconceptualization of the 'negative staff attitudes and poor care in nursing home' assumption. Gerontologist 1988; 28: 813-820.

12. Holmes D, Teresi T. Personnel costs in special dementia care units compared with costs on traditional care units. Special Care Units 2000; 4: 199-215.

13. Mehr D, Fries B. Resource use on Alzheimer's Special Care Units. Gerontologist 1995; 35: 179-184.

14. Fries B, Mehr D, Schneider D, Foley W, Burke R. Mental dysfunction and resource use in nursing homes. Med Care 1993; 31 (10): 898-920.

15. Fox D. What are the Costs of AIDS: Alternative Methodological Approaches. Health Services Research Methodology: A Focus on AIDS, Conference proceedings. National Center for Health Services Research and Health Technology Assessment, Public Health Service, US DHHS, 1989.

16. Scitovsky A. Past lessons and future directions: The economics of health services delivery for HIV-related illnesses. In New Perspectives on HIV-Related Illnesses: Progress in Health Services Research, LeVee W (ed.), Report No. (PHS) 89-3449. NTIS: Springfield, VA, 1989.

17. Seage G. New Perspectives on HIV-Related Illnesses: Progress in Health Services Research, LeVee W (ed.), discussion paper Report No. (PHS) 89-3449. NTIS, Springfield, VA, 1989.

18. Hildalgo J. Collecting statewide data on HIV costs and financing: pitfalls and payoffs. In New Perspectives on HIV-Related Illnesses: Progress in Health Services Research, LeVee W (ed.), Report No. (PHS) 89-3449.

19. Holmes D, Teresi J, Lindeman D, Glandon G. Measurement of personal care inputs in chronic care settings. J Mental Health Aging 1997; 3: 119-127.

20. Holmes D, Teresi J, Ramirez M, Goldman D. The measurement and comparison of staff service inputs in special dementia care units and in traditional nursing home units using a barcode methodology. $J$ Mental Health Aging 1998; 3: 195-207.

21. Folstein MF, Folstein SE, McHugh PR. Mini-Mental State: A practical guide for grading the cognitive state of patients for the clinician. $J$ Psychiatric Res 1975; 12: 189-198.

22. SPSS, Inc. SPSS/PC + Advanced Statistics 2.0. Chicago, IL, 1988

23. Ory M. Commentary: Dementia Special Care: The Development of a National Research Initiative. Alzheimer Dis Assoc Disorders 1994; 8: $389-394$.

24. Gurland B, Kuriansky J, Sharpe L, Simon R, Stiller P, Birkett P. The Comprehensive Assessment and Referral Evaluation (CARE):Rationale, development and reliability. Int J Aging Human Dev 197778; 8 (1): 9-42.

25. Teresi J, Golden R, Gurland B, Wilder D, Bennett R. Construct validity of indicator-scales developed for the Comprehensive Assessment and Referral Evaluation interview schedule. J Gerontol 1984; 39 (2): 147-157.

26. Teresi J, Golden R, Gurland B. Concurrent and predictive validity of indicator-scales developed for the Comprehensive Assessment and Referral Evaluation interview schedule. J Gerontol 1984; 39 (2): $158-165$.

27. Mann AH, Wood K, Cross P, Gurland B, Schreiber P, Haefner H. Institutional care of the elderly: A comparison of the cities of New York and London and Mannheim. Soc Psychiatry 1984; 19: 1-6.

28. Holmes D. Direct Behavior Observation Methods for Studying Special Care Units for Alzheimer's Patients: Use of the InfoAide Barcode System. Symposium participant at the Annual Meetings of the Gerontological Society of America, Atlanta, 1994.

29. Holmes D, Lindeman D, Ory M, Teresi J. Measurement of service units and costs of care for persons with dementia in special care units. Alzheimer's Dis Assoc Disorders: Int J 1994; 8: S328-S340.

30. Laird NM, Ware JH. Random effects model for longitudinal data. Biometrics 1984; 44: 175-188.

31. Littell RC, Milliken GA, Stroup WW, Wolfinger RD. SAS System for Mixed Models. Gary, NC: SAS.

32. van Haitsma K, Lawton MP, Kleban MH. Does segregation help or hinder?: Examining the role of homogeneity in behavioral and emotional aspects of quality of life for persons with cognitive impairment in the nursing home. Res Practice Alzheimer's Dis 2000; 4: 163-177. 